\title{
Preliminary Assessment of Suomi-NPP VIIRS On-orbit Radiometric Performance
}

Hassan Oudrari, Frank De Luccia, Jeff Mcintire, David Moyer, Vincent Chiang, Xiaoxiong Xiong, Jim Butler

\section{Long Abstract:}

The Visible-Infrared Imaging Radiometer Suite (VIIRS) is a key instrument on-board the Suomi National Polar-orbiting Partnership (NPP) spacecraft that was launched on October $28^{\text {th }} 2011$. VIIRS was designed to provide moderate and imaging resolution of most of the globe twice daily. It is a wide-swath $(3,040 \mathrm{~km})$ cross-track scanning radiometer with spatial resolutions of 370 and $740 \mathrm{~m}$ at nadir for imaging and moderate bands, respectively. It has 22 spectral bands covering the spectrum between $0.412 \mu \mathrm{m}$ and $12.01 \mu \mathrm{m}$, including 14 reflective solar bands (RSB), 7 thermal emissive bands (TEB), and 1 day-night band (DNB). VIIRS observations are used to generate 22 environmental data products (EDRs). This paper will briefly describe NPP VIIRS calibration strategies performed by the independent government team, for the initial on-orbit Intensive Calibration and Validation (ICV) activities. In addition, this paper will provide an early assessment of the sensor on-orbit radiometric performance, such as the sensor signal to noise ratios (SNRs), dual gain transition verification, dynamic range and linearity, reflective bands calibration based on the solar diffuser (SD) and solar diffuser stability monitor (SDSM), and emissive bands calibration based on the on-board blackbody calibration (OBC). A comprehensive set of performance metrics generated during the pre-launch testing program will be compared to VIIRS on-orbit early performance, and a plan for future cal/val activities and performance enhancements will be presented.

\section{Short Abstract:}

The Visible-Infrared Imaging Radiometer Suite (VIIRS) is a key instrument on-board the Suomi National Polar-orbiting Partnership (NPP) spacecraft that was launched on October $28^{\text {th }} 2011$. VIIRS has 22 spectral bands covering the spectrum between $0.412 \mu \mathrm{m}$ and $12.01 \mu \mathrm{m}$. This paper will briefly describe NPP VIIRS calibration strategies performed for the initial on-orbit Intensive Calibration and Validation (ICV) activities, and will provide an overview of the VIIRS pre-launch characterization and calibration testing and assessments, and an early assessment of the sensor on-orbit radiometric performance. Planning for future cal/val activities for performance enhancements will also be presented. 